\title{
Acute painful peripheral neuropathy due to metronidazole
}

Sir,

Metronidazole is a 5-nitroimidazole compound that has potent activity against anaerobic bacteria and several protozoa. Peripheral neuropathy is its rare side effect. ${ }^{[1]}$ We report an unusually rapid development of peripheral neuropathy due to metronidazole. A 45-year-old lady received oral metronidazole for the treatment of vaginitis. After 3 days of treatment, after receiving $3.6 \mathrm{~g}$ of metronidazole, she developed severe burning pain in both the feet and aching pain in the muscles of the thighs and calves. On the seventh day she developed burning pain in the hands and fingers and severe aching pain in the muscles of forearms and arms. There were no history of diabetes mellitus, chronic alcohol intake, renal failure, occupational toxin exposure or chronic diarrhoea. She consumed wholesome non-vegetarian diet. There was no history of arthritis, skin rash, recurrent oral ulcers, uveitis, xerostomia or xeropthalmia.

Examination revealed a well-nourished middle-aged lady. There was no pallor, hyper-pigmentation, and evidence of nutritional deficiencies or hypopigmented skin lesions. She weighed $65 \mathrm{~kg}$ and her height was $154 \mathrm{~cm}$. Her blood pressure was 120/ $70 \mathrm{~mm} \mathrm{Hg}$. She had no organomegaly. Detailed neurological examination was normal. Her hemogram, peripheral smear, fasting blood sugar, serum creatinine and serum vitamin B12 level were normal. Antinuclear antibodies were negative.

Electrophysiological studies done on the $10^{\text {th }}$ day of illness revealed prolonged distal motor latency $(6.5 \mathrm{~ms}$ for a distance of $90 \mathrm{~mm}$ ) of posterior tibial nerve, prolonged distal motor latency $(5.3 \mathrm{~ms}$ for a distance of $90 \mathrm{~mm}$ ) and mildly reduced compound muscle action potential amplitude $(3.1 \mathrm{mV})$ of the peroneal nerve and decreased sensory nerve action potential amplitude $(2.0 \mathrm{mV})$ of the posterior tibial nerve. Nerve conduction studies of median, ulnar and sural nerves were normal. Metronidazole was discontinued and symptomatic therapy (Carbamazepine and Gabapentin) was given. She found significant relief of symptoms. After 3 months, she continued to take carbamazepine and gabapentin for symptomatic relief. A repeat nerve conduction study at 3 months did not show any improvement in the abnormalities. Applying the Naranjo's algorithm, ${ }^{[2]}$ our patient's neuropathy could be considered a "probable (score +5$)$ " adverse effect of metronidazole.

The cumulative neurotoxic dose of metronidazole in the lit- 
erature varied from 13.2 grams $^{[3]}$ to 228 grams. $^{[4]}$ The duration of therapy after which neuropathic symptoms developed varied from 11 days $^{[3]}$ to 6 months. ${ }^{[4]}$ The 3 days of latency and $3.6 \mathrm{~g}$ of cumulative dose observed in our patient are the smallest reported so far. It is noteworthy that, in the case reports from India, ${ }^{[3,5,]}$ the cumulative dose of metronidazole was low (13.2-18 grams) and the latency to symptom onset very short (11 days to 18 days) when compared to patients from the West. This may reflect a genetic susceptibility to the neurotoxic effects of metronidazole or a genetic variation in the metabolism of metronidazole in Indian patients.

To conclude, we report an unusually rapid development of peripheral neuropathy after starting metronidazole. Its early recognition and rapid withdrawal of the drug are important, as the neuropathy can be disabling and persistent.

\section{G. R. K. Sarma, V. Kamath}

Dept. of Neurology St. Johnæs Medical college hospital Bangalore 560034, India, E-mail: grk_sarma@yahoo.com

\section{References}

1. Rustscheff S, Hulten S. An unexpected and severe neurological disorder with permanent disability aquired during short course treatment with metronidazole. Scand J Infect Dis 2003;53:279-80.

2. Naranjo CA, Busto U, Sellers EM, Sandor P, Ruiz I, Roberts EA, et al. A method for estimating the probability of adverse drug reactions. Clin Pharmacol Ther 1981;30:239-45.

3. Pais P, Balasubramaniam KR Metronidazole-peripheral neuropathy. (Letter to Editor). JAPI 1982;30:918-9.

4. Bradley WG, Karlson IJ, Rassol CG. Metronidazole neuropathy. Br Med J 1977;2:610-11.

5. Kapoor K, Chandra M, Nag D, Paliwal JK, Gupta RC, Saxena RC. Evaluation of metronidazole toxicity: a prospective study. Int J Clin Pharmacol Res $1999 ; 19: 3: 83-8$. 